\title{
Effective interaction between monolayers of block copolymer compatiblizer in a polymer blend
}

Article

Published Version

Thompson, R. B. and Matsen, M. W. (2000) Effective interaction between monolayers of block copolymer compatiblizer in a polymer blend. The Journal of Chemical Physics, 112 (15). 6863. ISSN 0021-9606 doi: https://doi.org/10.1063/1.481262 Available at https://centaur.reading.ac.uk/63617/

It is advisable to refer to the publisher's version if you intend to cite from the work. See Guidance on citing.

Published version at: http://dx.doi.org/10.1063/1.481262

To link to this article DOI: http://dx.doi.org/10.1063/1.481262

Publisher: American Institute of Physics

All outputs in CentAUR are protected by Intellectual Property Rights law, including copyright law. Copyright and IPR is retained by the creators or other copyright holders. Terms and conditions for use of this material are defined in the End User Agreement.

www.reading.ac.uk/centaur 
Central Archive at the University of Reading

Reading's research outputs online 


\section{AP $\begin{aligned} & \text { The Journal of } \\ & \text { Chemical Physics }\end{aligned}$}

\section{Effective interaction between monolayers of block copolymer compatiblizer in a polymer blend}

R. B. Thompson and M. W. Matsen

Citation: The Journal of Chemical Physics 112, 6863 (2000); doi: 10.1063/1.481262

View online: http://dx.doi.org/10.1063/1.481262

View Table of Contents: http://scitation.aip.org/content/aip/journal/jcp/112/15?ver=pdfcov

Published by the AIP Publishing

\section{Articles you may be interested in}

Orientational interactions in block copolymer melts: Self-consistent field theory

J. Chem. Phys. 137, 104911 (2012); 10.1063/1.4752198

Interaction between a rodlike inclusion and a supported bilayer membrane

J. Chem. Phys. 125, 164710 (2006); 10.1063/1.2359436

Nucleation in binary polymer blends: Effects of adding diblock copolymers

J. Chem. Phys. 118, 8997 (2003); 10.1063/1.1566941

Mean-field studies of block copolymer/homopolymers blends

AIP Conf. Proc. 519, 247 (2000); 10.1063/1.1291561

Elastic properties of a diblock copolymer monolayer and their relevance to bicontinuous microemulsion

J. Chem. Phys. 110, 4658 (1999); 10.1063/1.478349

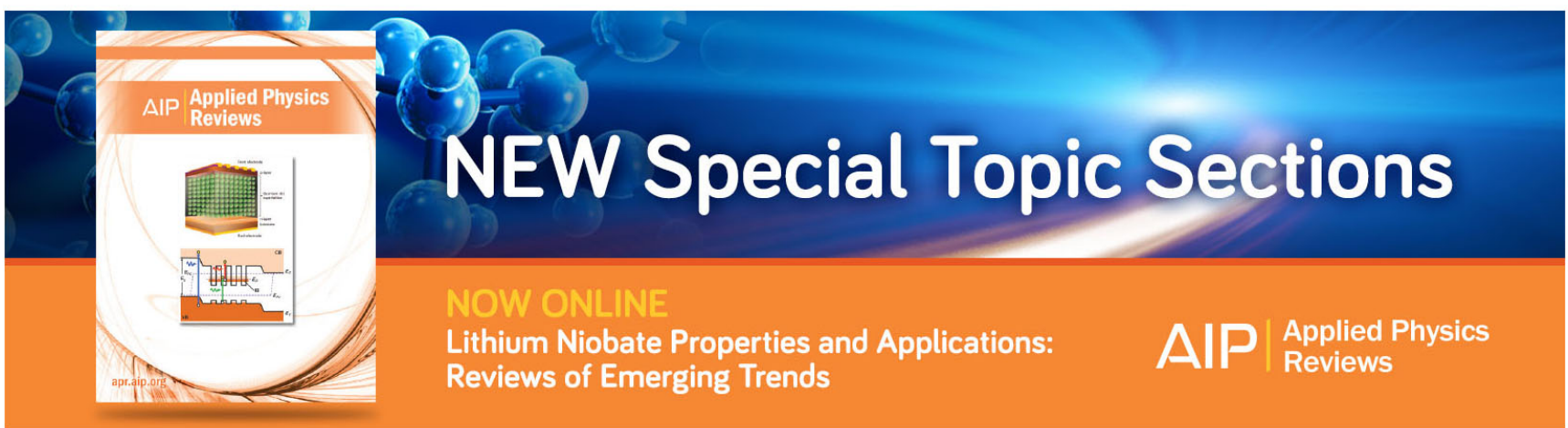




\title{
Effective interaction between monolayers of block copolymer compatiblizer in a polymer blend
}

\author{
R. B. Thompson and M. W. Matsen ${ }^{\text {a) }}$ \\ Polymer Science Centre, University of Reading, Whiteknights, Reading RG6 6AF, United Kingdom
}

(Received 12 October 1999; accepted 27 January 2000)

\begin{abstract}
The stability of ternary blends of two immiscible homopolymers and a block copolymer compatiblizer depends crucially on the effective interaction between the copolymer monolayers that form between the unlike homopolymer domains. Here, the interaction is calculated for blends involving $A$ and $B$ homopolymers of equal size with $A B$ diblock copolymers of symmetric composition using both self-consistent field theory (SCFT) and strong-segregation theory (SST). If the homopolymers are larger than the copolymer molecules, an attractive interaction is predicted which would destroy the blend. This conclusion coupled with considerations regarding the elastic properties of the monolayer suggests that the optimum size of the homopolymer molecules is about $80 \%$ that of the copolymer molecule. A detailed examination of the theory demonstrates that the attraction results from the configurational entropy loss of the homopolymer molecules trapped between the copolymer monolayers. We conclude by suggesting how the monolayers can be altered in order to suppress this attraction and thus improve compatiblization. (C) 2000 American Institute of Physics. [S0021-9606(00)50615-1]
\end{abstract}

\section{INTRODUCTION}

Polymeric alloys provide a tremendous opportunity to create new materials with properties that are easily tailored by adjusting the blend composition. Unfortunately, most homopolymer blends are unstable, and consequently the homopolymer components macrophase separate into their own domains. Although mechanical blending can produce small homopolymer domains, these domains will begin to grow (i.e., coarsen) after the mixing ceases, and as that happens the mechanical properties of the alloy will deteriorate. One solution to this problem has been to add block copolymer, where the immiscible components are combined into a single molecule. ${ }^{1,2}$ The copolymer molecules act as amphiphiles forming a thin monolayer between the immiscible homopolymer domains. This, first of all, improves the adhesion between the different domains and thus the mechanical properties of the alloy. Second, it greatly reduces the interfacial tension and hence the driving force towards macrophase separation. Third, the monolayers produce a steric repulsion that inhibits the coalescence of domains. Despite the block copolymer, polymeric blends generally remain unstable, and thus the homopolymer domains still coarsen although far more slowly. If the kinetics are sufficiently slow, it is still possible to produce effective polymeric alloys. Better yet, the block copolymer can, under appropriate conditions, produce a stable blend called a microemulsion, ${ }^{3-5}$ in which the homopolymer domains do not coarsen.

The ability of block copolymer to form a useful blend depends crucially on the effective interaction between the monolayers. For example, the steric repulsion that results when two monolayers come into close contact impedes the coalescence of domains, which is an important mechanism in

${ }^{\text {a) }}$ Author to whom correspondence should be addressed. the coarsening of polymer blends. ${ }^{1,6}$ Also important is the interaction at intermediate distances where, in fact, an attraction can occur. Experiments ${ }^{7}$ have found this to be a problem when the homopolymer molecules become large relative to the copolymer molecules. The attraction causes the copolymer monolayers to stick together, forcing out the two homopolymers, which then macrophase separate. Although this problem can be avoided by using large copolymer molecules, doing so has the drawback of producing stiff monolayers. ${ }^{8}$ In order to produce stable microemulsions with microscopic domains, the monolayers have to be flexible, ${ }^{9}$ and consequently the block copolymer molecules have to be small. ${ }^{8}$ Therefore, the optimum block copolymer compatiblizer will be the smallest one that does not cause a significant attraction between the monolayers.

The crossover from a repulsive to an attractive interaction with increasing homopolymer size has been modeled previously for two very similar systems. Shull ${ }^{10}$ has examined the interaction between two polymer brushes separated by a chemically identical homopolymer matrix using selfconsistent field theory (SCFT). Semenov ${ }^{11}$ has examined the interaction between $A B$ diblock copolymer bilayers in an $A$ homopolymer matrix using strong-segregation theory (SST). Both studies attribute the effective interaction to the entropic energy of the homopolymer. Its translational entropy produces a repulsion while its configurational entropy causes an attraction. The former contribution is dominant when the homopolymer molecules are small, where as the latter is more important at high molecular weights. These same mechanisms are also present in polymerically stabilized colloidal dispersions. ${ }^{12}$ However, to our knowledge, this effect has not yet been theoretically examined in ternary blends of two immiscible homopolymers with a block copolymer compatiblizer. 
Below, we provide a detailed theoretical examination of the interaction between monolayers of symmetric $A B$ diblock copolymer in a blend of equally sized $A$ and $B$ homopolymers. To provide accurate predictions for designing actual polymeric microemulsions, we use the SCFT of Helfand, ${ }^{13}$ which is the state of the art in block copolymer theory. Our conclusion is that the optimum homopolymer to copolymer size ratio is about 0.8 . The SCFT results are also compared to those using the SST of Semenov. ${ }^{14}$ Although the SST is found to be considerably inaccurate, it is useful for developing an intuitive explanation for the resulting interaction. By closely examining the various free-energy contributions to the interaction using both SCFT and SST, we provide definitive evidence supporting the previous explanation by Shull ${ }^{10}$ and Semenov. ${ }^{11}$ Based on this thorough explanation, we conclude by suggesting how the compatiblizer can be altered in order to produce superior polymeric microemulsions.

\section{SELF-CONSISTENT FIELD THEORY}

In this section, we formulate the $\mathrm{SCFT}^{8,13,15,16}$ for a pair of $A B$ diblock copolymer monolayers in an immiscible blend of $A$ - and $B$-rich homopolymer phases. Each diblock molecule is composed of $N$ segments of which a fraction $f$ forms the A block, and each $\nu$-type homopolymer is composed of $N \alpha_{h \nu}$ segments $(\nu=A$ or $B)$. The $A$ and $B$ segments are assumed to be incompressible and are defined based on a common segment volume, $\rho_{0}^{-1}$. The segments are also assumed to be completely flexible with statistical lengths, $a_{A}$ and $a_{B}$, respectively, ${ }^{17}$ i.e., the unperturbed rms end-to-end length of the diblock molecule is $a N^{1 / 2}$, where $a \equiv\left[f a_{A}^{2}\right.$ $\left.+(1-f) a_{B}^{2}\right]^{1 / 2}$. The interaction between $A$ and $B$ segments is controlled by the usual Flory-Huggins $\chi$ parameter.

The geometry of our system is illustrated in Fig. 1(a). We consider a system of finite width in the $z$ direction (i.e., $-\Delta \leqslant z \leqslant \Delta$ ) with two flat monolayers, each of area $\mathcal{A}$, positioned perpendicular to the $z$ axis at $z= \pm d / 2$. An $A$-rich homopolymer region exists between the monolayers provided $d$ is not too small, and $B$-rich homopolymer regions occur on the outer sides. We choose $\Delta$ large enough that the B-rich regions attain bulk conditions by $z= \pm \Delta$; generally, $\Delta / a N^{1 / 2}=3$ is sufficient. Below the schematic diagram in Fig. 1 are plots of the dimensionless segment concentrations $\phi_{c \nu}(z)$ and $\phi_{h \nu}(z)(\nu=A$ or $B)$ of the copolymer and homopolymer molecules, respectively, calculated using SCFT and SST at $\chi N=100, \alpha_{h A}=\alpha_{h B}=1$, and $d / a N^{1 / 2}=2$.

In SCFT, the molecular interactions are represented by two static fields: $w_{A}(z)$ acts on $A$ segments and $w_{B}(z)$ acts on $B$ segments. These fields are given by

$$
w_{A}(z)=\chi N \phi_{B}(z)+\xi(z)-\psi \Delta[\delta(z-d / 2)+\delta(z+d / 2)],
$$

$$
w_{B}(z)=\chi N \phi_{A}(z)+\xi(z)+\psi \Delta[\delta(z-d / 2)+\delta(z+d / 2)],
$$

where $\phi_{\nu}(z)=\phi_{c \nu}(z)+\phi_{h \nu}(z)$. In each case, the first term represents the actual segment interactions. The next term is a Lagrange multipler, $\xi(z)$, used to enforce the incompressibility constraint, (a)

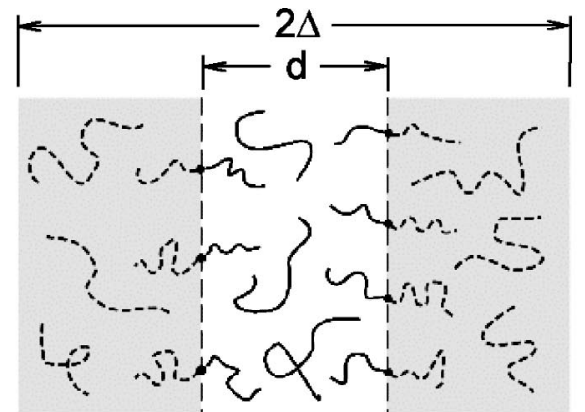

(b)

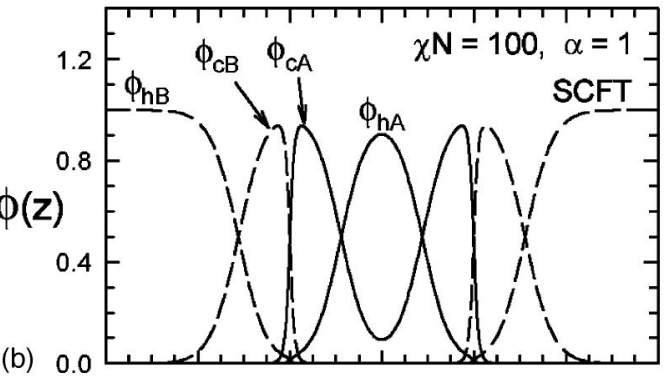

(c)

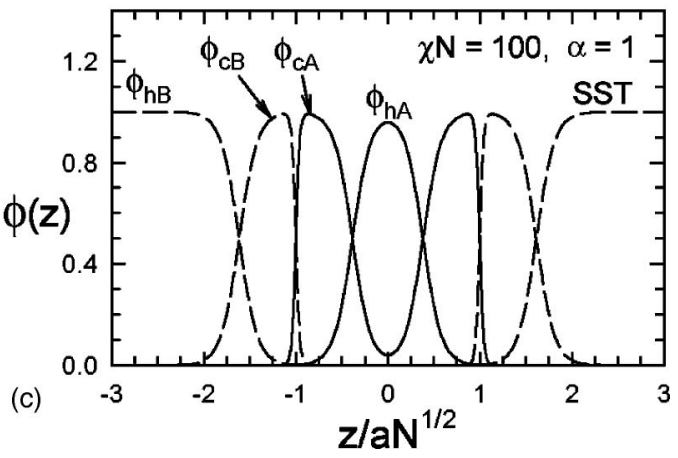

FIG. 1. (a) Schematic diagram of two $A B$ diblock copolymer monolayers separated by a distance, $d$, with $A$-type homopolymer between and $B$-type homopolymer to the outside. (For calculation purposes, we consider a system of finite width, $2 \Delta$.) The position of the interfaces denoted by vertical dotted lines is defined as the point where the total $A$ - and $B$-segment concentrations are equal [i.e., $\left.\phi_{A}(z)=\phi_{B}(z)\right]$. The plots below show the segment profiles for the $A$ brush $\phi_{c A}(z)$, the $B$ brush $\phi_{c B}(z)$, the $A$ homopolymer $\phi_{h A}(z)$, and the $B$ homopolymer $\phi_{h B}(z)$ calculated at $\chi N=100$ and $\alpha_{h A}=\alpha_{h B} \equiv \alpha=1.0$ using (b) SCFT and (c) SST. Solid and dashed curves represent $A$ - and $B$-segment quantities, respectively.

$$
\phi_{A}(z)+\phi_{B}(z)=1 .
$$

The last term contains another Lagrange multiplier, $\psi$, used to fix the location of the two interfaces by enforcing the condition

$$
\phi_{A}( \pm d / 2)=\phi_{B}( \pm d / 2) \text {. }
$$

Introducing the fields allows us to perform the statistical mechanics for each of the three different molecules. For a diblock copolymer molecule, we parametrize its contour by $s$, where $s=0$ at the $A$ end, $s=f$ at the junction, and $s=1$ at the $B$ end. Then we fix a segment $s$ at some location with coordinate $z$, and calculate the partial partition function, $q_{c}(z, s)$, for the $(0, s)$ portion of the chain. This quantity satisfies the modified diffusion equation, 


$$
\frac{\partial}{\partial s} q_{c}(z, s)= \begin{cases}{\left[\frac{N a_{A}^{2}}{6} \frac{\partial^{2}}{\partial z^{2}}-w_{A}(z)\right] q_{c}(z, s),} & \text { if } 0<s<f, \\ {\left[\frac{N a_{B}^{2}}{6} \frac{\partial^{2}}{\partial z^{2}}-w_{B}(z)\right] q_{c}(z, s),} & \text { if } f<s<1,\end{cases}
$$

with the initial condition $q_{c}(z, 0)=1 .^{13}$ The partial partition function, $q_{c}^{\dagger}(z, s)$, for the $(s, 1)$ portion of the chain is calculated by solving the same differential equation except with the right-hand side multiplied by -1 and the condition $q_{c}^{\dagger}(z, 1)=1$. Similarly, we parametrize each $\nu$-type homopolymer with $0<s<\alpha_{h \nu}$, and fix the location of a segment $s$. The partial partition function for the $(0, s)$ portion of the homopolymer satisfies

$$
\frac{\partial}{\partial s} q_{h \nu}(z, s)=\left[\frac{N a_{\nu}^{2}}{6} \frac{\partial^{2}}{\partial z^{2}}-w_{\nu}(z)\right] q_{h \nu}(z, s),
$$

with $q_{h \nu}(z, 0)=1$. Because the two ends of a homopolymer molecule are identical, the partial partition function of the $\left(s, \alpha_{h \nu}\right)$ portion is just $q_{h \nu}\left(z, \alpha_{h \nu}-s\right)$.

In terms of the above functions, the complete partition function of a single copolymer molecule in the fields is

$$
\mathcal{Q}_{c}=\mathcal{A} \int_{-\Delta}^{\Delta} d z q_{c}(z, s) q_{c}^{\dagger}(z, s),
$$

which can be evaluated for any point $s$ on the chain. Similarly, the full partition function of a $\nu$-type homopolymer is

$$
\mathcal{Q}_{h \nu}=\mathcal{A} \int_{-\Delta}^{\Delta} d z q_{h \nu}(z, s) q_{h \nu}\left(z, \alpha_{h \nu}-s\right) .
$$

The partial partition functions are also used to calculate the segment profiles: ${ }^{15}$

$$
\begin{aligned}
& \phi_{c A}(z)=\int_{0}^{f} d s q_{c}(z, s) q_{c}^{\dagger}(z, s), \\
& \phi_{c B}(z)=\int_{f}^{1} d s q_{c}(z, s) q_{c}^{\dagger}(z, s), \\
& \phi_{h \nu}(z)=z_{h \nu} \int_{0}^{\alpha_{h \nu}} d s q_{h \nu}(z, s) q_{h \nu}\left(z, \alpha_{h \nu}-s\right),
\end{aligned}
$$

where $z_{h \nu} \equiv \exp \left(\mu_{h \nu} / k_{B} T\right)$ and $\mu_{h \nu}$ is the chemical potential controlling the concentration of $\nu$-type homopolymer ( $\nu$ $=A$ or $B)$. Because of the incompressibility constraint, Eq. (3), there is no need to introduce a chemical potential to control the copolymer concentration.

Now that we have the concentrations, the fields and the Lagrange multipliers can be adjusted by a quasi-NewtonRaphson method so as to satisfy Eqs. (1)-(4). After that is accomplished, the free energy of the system, $F$, is given by

$$
\begin{aligned}
\frac{N F}{k_{B} T \rho_{0}}= & -\mathcal{Q}_{c}-z_{h A} \mathcal{Q}_{h A}-z_{h B} \mathcal{Q}_{h B} \\
& +\mathcal{A} \int_{-\Delta}^{\Delta} d z\left[\chi N \phi_{A}(z) \phi_{B}(z)\right. \\
& \left.-w_{A}(z) \phi_{A}(z)-w_{B}(z) \phi_{B}(z)\right] .
\end{aligned}
$$

Rather than solving the above equations in real space, we use a Fourier method analogous to that in Ref. 8.

In addition to solving these equations for the geometry in Fig. 1(a), we must also solve them for the bulk homopolymer-rich phases. In these uniform phases, none of the quantities depend on $z$, and thus the equations simplify immensely. ${ }^{15}$ The free energy, $F^{(\nu)}$, for a volume, $\mathcal{V}_{\nu}$, of the $\nu$-rich homopolymer phase is expressed as

$$
\begin{aligned}
\frac{N F^{(\nu)}}{k_{B} T \rho_{0} \mathcal{V}_{\nu}}= & \frac{\phi_{h A}^{(\nu)}}{\alpha_{h A}}\left(\ln \frac{\phi_{h A}^{(\nu)}}{\alpha_{h A}}-1\right)+\frac{\phi_{h B}^{(\nu)}}{\alpha_{h B}}\left(\ln \frac{\phi_{h B}^{(\nu)}}{\alpha_{h B}}-1\right) \\
& +\phi_{c}^{(\nu)}\left(\ln \phi_{c}^{(\nu)}-1\right)+\chi N\left(\phi_{h A}^{(\nu)}+f \phi_{c}^{(\nu)}\right)\left(\phi_{h B}^{(\nu)}\right. \\
& \left.+(1-f) \phi_{c}^{(\nu)}\right)-\frac{\mu_{h A} \phi_{h A}^{(\nu)}}{\alpha_{h A} k_{B} T}-\frac{\mu_{h B} \phi_{h B}^{(\nu)}}{\alpha_{h B} k_{B} T},
\end{aligned}
$$

where the copolymer concentration satisfies $\phi_{c}^{(\nu)}=1-\phi_{h A}^{(\nu)}$ $-\phi_{h B}^{(\nu)}$, and the homopolymer concentrations, $\phi_{h \nu}^{(\nu)}$, are determined by

$$
\begin{aligned}
\frac{\mu_{h A}}{\alpha_{h A} k_{B} T}= & \frac{1}{\alpha_{h A}} \ln \frac{\phi_{h A}^{(\nu)}}{\alpha_{h A}}-\ln \phi_{c}^{(\nu)}-\chi N(1-f)\left(\phi_{h A}^{(\nu)}-\phi_{h B}^{(\nu)}\right. \\
& \left.-(1-2 f) \phi_{c}^{(\nu)}\right), \\
\frac{\mu_{h B}}{\alpha_{h B} k_{B} T}= & \frac{1}{\alpha_{h B}} \ln \frac{\phi_{h B}^{(\nu)}}{\alpha_{h B}}-\ln \phi_{c}^{(\nu)}+\chi N f\left(\phi_{h A}^{(\nu)}-\phi_{h B}^{(\nu)}\right. \\
& \left.-(1-2 f) \phi_{c}^{(\nu)}\right) .
\end{aligned}
$$

The chemical potentials, $\mu_{h A}$ and $\mu_{h B}$, must be adjusted so that the $A$ - and $B$-rich homopolymer phases are in coexistence (i.e., $F^{(A)} / \mathcal{V}_{A}=F^{(B)} / \mathcal{V}_{B}$ ).

For the system in Fig. 1(a), the volume of the $A$-rich region is $\mathcal{V}_{A}=\mathcal{A} d$ and the $B$-rich volume is $\mathcal{V}_{B}=\mathcal{A}(2 \Delta$ $-d$ ), where $\mathcal{A}$ is the area of each interface. The interfacial excess $X^{(\mathrm{ex})}$ of a quantity $X$ is then defined as

$$
\frac{X^{(\mathrm{ex})}}{\mathcal{A}} \equiv 2 \Delta \frac{X}{\mathcal{V}}-d \frac{X^{(A)}}{\mathcal{V}_{A}}-(2 \Delta-d) \frac{X^{(B)}}{\mathcal{V}_{B}}
$$

From the excess free energy, $F^{(\mathrm{ex})}$, the interaction energy between the two monolayers is given by

$$
V(d)=\frac{F^{(\mathrm{ex})}}{\mathcal{A}}-2 \sigma,
$$

where $\sigma$ is the interfacial tension. (The factor of 2 occurs because there are two interfaces.) In our study, we consider saturated interfaces where the chemical potentials, $\mu_{h A}$ and $\mu_{h B}$, are adjusted so that $\sigma=0$.

In order to identify the source of the effective interaction between the monolayers, the free energy can be separated into various physically relevant contributions. We start by decomposing it as

$$
F=U-T\left(S_{c}+S_{h A}+S_{h B}\right)-\mu_{h A} n_{h A}-\mu_{h B} n_{h B},
$$

where $U$ is the internal energy, $S_{c}$ is the copolymer entropy, $S_{h A}$ is the $A$-homopolymer entropy, $S_{h B}$ is the $B$-homopolymer entropy, $n_{h A}$ is the number of $A$ homopolymers, and $n_{h B}$ is the number of $B$ homopolymers. These contributions to $F$ are given by 


$$
\begin{gathered}
\frac{N U}{k_{B} T \rho_{0} \mathcal{V}}=\frac{\chi N}{2 \Delta} \int_{-\Delta}^{\Delta} d z \phi_{A}(z) \phi_{B}(z) \\
-\frac{N S_{c}}{k_{B} \rho_{0} \mathcal{V}}=\bar{\phi}_{c}\left[\ln \left(\frac{\mathcal{V} \bar{\phi}_{c}}{\mathcal{Q}_{c}}\right)-1\right]-\frac{1}{2 \Delta} \int_{-\Delta}^{\Delta} d z\left[w_{A}(z) \phi_{c A}(z)\right. \\
\left.+w_{B}(z) \phi_{c B}(z)\right] \\
-\frac{N S_{h \nu}}{k_{B} \rho_{0} \mathcal{V}}=\frac{\bar{\phi}_{h \nu}}{\alpha_{h \nu}}\left[\ln \left(\frac{\mathcal{V} \bar{\phi}_{h \nu}}{\mathcal{Q}_{h \nu} \alpha_{h \nu}}\right)-1\right] \\
-\frac{1}{2 \Delta} \int_{-\Delta}^{\Delta} d z \quad w_{\nu}(z) \phi_{h \nu}(z) \\
-\frac{N \mu_{h \nu} n_{h \nu}}{k_{B} T \rho_{0} \mathcal{V}}=-\frac{\mu_{h \nu} \bar{\phi}_{h \nu}}{k_{B} T \alpha_{h \nu}}
\end{gathered}
$$

where the volume-averaged copolymer and homopolymer concentrations are

$$
\begin{aligned}
& \bar{\phi}_{c} \equiv \frac{1}{2 \Delta} \int_{-\Delta}^{\Delta} d z \phi_{c}(z)=\frac{N n_{c}}{\rho_{0} \mathcal{V}} \\
& \bar{\phi}_{h \nu} \equiv \frac{1}{2 \Delta} \int_{-\Delta}^{\Delta} d z \quad \phi_{h \nu}(z)=\frac{N \alpha_{h \nu} n_{h \nu}}{\rho_{0} \mathcal{V}} .
\end{aligned}
$$

This is not a sufficient break down of $F$; we must go one step further and separate the entropy of the homopolymer into

$$
S_{h \nu}=S_{h \nu, \text { trans }}+S_{h \nu, \text { conf }},
$$

where the translational entropy $S_{h \nu \text {,trans }}$ and the configurational entropy $S_{h \nu, \text { conf }}$ are given by

$$
\begin{aligned}
-\frac{N S_{h \nu, \text { trans }}}{k_{B} \rho_{0} \mathcal{V}}= & \frac{1}{2 \Delta} \int_{-\Delta}^{\Delta} d z \rho_{C, h \nu}(z)\left[\ln \rho_{C, h \nu}(z)-1\right] \\
-\frac{N S_{h \nu, \text { conf }}}{k_{B} \rho_{0} \mathcal{V}}= & -\frac{1}{2 \Delta} \int_{-\Delta}^{\Delta} d z\left[2 \rho_{C, h \nu}(z) \ln q_{h \nu}\left(z, \alpha_{h \nu} / 2\right)\right. \\
& \left.+w_{\nu}(z) \phi_{h \nu}(z)\right] .
\end{aligned}
$$

These expressions involve the central segment (i.e., $s$ $\left.=\alpha_{h \nu} / 2\right)$ distributions of the homopolymers,

$$
\rho_{C, h \nu}(z)=\frac{\mathcal{V} q_{h \nu}^{2}\left(z, \alpha_{h \nu} / 2\right) \bar{\phi}_{h \nu}}{\mathcal{Q}_{h \nu} \alpha_{h \nu}},
$$

which for convenience have been normalized such that their volume averages satisfy $\bar{\rho}_{C, h \nu}=\bar{\phi}_{h \nu} / \alpha_{h \nu}$.

In the $A$-rich phase, where all the concentrations are constant, the free energy decomposition reduces to

$$
\begin{aligned}
& \frac{N U^{(A)}}{k_{B} T \rho_{0} \mathcal{V}_{A}}=\chi N\left(f \phi_{c}^{(A)}+\phi_{h A}^{(A)}\right)\left((1-f) \phi_{c}^{(A)}+\phi_{h B}^{(A)}\right), \\
& -\frac{N S_{c}^{(A)}}{k_{B} \rho_{0} \mathcal{V}_{A}}=\phi_{c}^{(A)}\left[\ln \phi_{c}^{(A)}-1\right], \\
& -\frac{N S_{h \nu, \text { trans }}^{(A)}}{k_{B} \rho_{0} \mathcal{V}_{A}}=\frac{\phi_{h \nu}^{(A)}}{\alpha_{h \nu}}\left[\ln \frac{\phi_{h \nu}^{(A)}}{\alpha_{h \nu}}-1\right]
\end{aligned}
$$

$$
-\frac{N S_{h \nu, \mathrm{conf}}^{(A)}}{k_{B} \rho_{0} \mathcal{V}_{A}}=0 .
$$

The expressions for the $B$-rich phase are analogous.

\section{STRONG-SEGREGATION THEORY}

Now we present a strong-segregation theory (SST) for the system illustrated in Fig. 1(a). This theory is an approximation to SCFT, where among other things it assumes that the diblock copolymers are highly stretched expelling most of the homopolymer thus producing reasonably dry monolayers. We closely follow the calculation of Semenov ${ }^{11}$ except that we work in the grand canonical ensemble, where the quantity of excess copolymer is free to adjust; this is important in order to capture some of the subtle effects present in the SCFT results and to properly model the steric repulsion between the monolayers.

As before, we separate the free energy, $F$, as in Eqs. (18) and (25). The expression for $U$, Eq. (19), remains unchanged. The entropic energy of the copolymer,

$$
\begin{aligned}
-\frac{N S_{c}}{k_{B} \rho_{0} \mathcal{V}}= & \frac{a^{2} N}{24 \Delta} \int_{-\Delta}^{\Delta} d z\left(\frac{\left[\phi_{c A, 1}^{\prime}(z)\right]^{2}}{\phi_{c A, 1}(z)}+\frac{\left[\phi_{c B, 1}^{\prime}(z)\right]^{2}}{\phi_{c B, 1}(z)}\right) \\
& +\frac{3 \pi^{2}}{8 f^{2} a^{2} N \Delta} \int_{-\Delta}^{\Delta} d z(z+d / 2)^{2}\left[\phi_{c A, 1}(z)\right. \\
& \left.+\phi_{c B, 1}(z)\right],
\end{aligned}
$$

is obtained for the brush profiles, $\phi_{c \nu, 1}(z)$, of the $z=-d / 2$ monolayer and then multiplied by two to account for the second monolayer. The first integral in Eq. (33) is the local configurational entropy loss due to variations in the segment profile and the second integral is the nonlocal stretching energy contribution. Each integral has separate terms for the $A$ and $B$ brushes. Here, we have assumed conformational symmetry (i.e., $a \equiv a_{A}=a_{B}$ ). The translational and configurational contributions to the entropic energy of the $\nu$-type homopolymer are

$$
\begin{aligned}
& -\frac{N S_{h \nu, \text { trans }}}{k_{B} \rho_{0} \mathcal{V}}=\frac{1}{2 \Delta} \int_{-\Delta}^{\Delta} d z \frac{\phi_{h \nu}(z)}{\alpha_{h \nu}}\left(\ln \frac{\phi_{h \nu}(z)}{\alpha_{h \nu}}-1\right), \\
& -\frac{N S_{h \nu, \text { conf }}}{k_{B} \rho_{0} \mathcal{V}}=\frac{a^{2} N}{48 \Delta} \int_{-\Delta}^{\Delta} d z \frac{\left[\phi_{h \nu}^{\prime}(z)\right]^{2}}{\phi_{h \nu}(z)},
\end{aligned}
$$

respectively. For $S_{h \nu \text {,trans }}$, we make the approximation $\rho_{C, h \nu}(z) \approx \phi_{h \nu}(z) / \alpha_{h \nu}$, which is accurate for small $\alpha_{h \nu}$ where this entropy term is actually important. ${ }^{11}$

In principle, the segment profiles should be obtained by minimizing the free energy, $F$. However, we follow Ref. 11 and assume hyperbolic tangent profiles. The interfacial widths $w$ and $w_{h \nu}$ of the $A / B$ interfaces and the copolymer/ homopolymer interfaces, respectively, will be determined by minimizing the free energy. Specifically, the $A$ brush profile at the $z=-d / 2$ interface is taken as

$$
\phi_{c A, 1}(z)=\frac{1}{2}\left[\tanh \left(\frac{2 z+d}{w}\right)-\tanh \left(\frac{2 z+d-2 h_{A}}{w_{h A}}\right)\right],
$$


where $h_{A}$ is the height of the $A$ brush. Similarly, the B brush profile at the same interface is

$$
\phi_{c B, 1}(z)=\frac{1}{2}\left[\tanh \left(\frac{2 z+d+2 h_{B}}{w_{h B}}\right)-\tanh \left(\frac{2 z+d}{w}\right)\right] .
$$

The brush profiles at the $z=d / 2$ interface are obtained by replacing $z$ with $-z$. The $A$ homopolymer profile is taken to be

$$
\phi_{h A}(z)=\frac{1}{2}\left[\tanh \left(\frac{2 z+d-2 h_{A}}{w_{h A}}\right)-\tanh \left(\frac{2 z-d+2 h_{A}}{w_{h A}}\right)\right],
$$

and the $B$ homopolymer profile is

$$
\begin{aligned}
\phi_{h B}(z)= & \frac{1}{2}\left[2-\tanh \left(\frac{2 z+d+2 h_{B}}{w_{h B}}\right)\right. \\
& \left.+\tanh \left(\frac{2 z-d-2 h_{B}}{w_{h B}}\right)\right] .
\end{aligned}
$$

The above profiles will only be accurate if the relative homopolymer sizes (i.e., $\alpha_{h A}$ and $\alpha_{h B}$ ) are large. Nevertheless, we use these simple functional forms because they allow many of the integrals to be evaluated analytically, which makes the SST results easier to interpret. Substituting $\phi_{\nu}(z)=\phi_{c \nu, 1}(z)+\phi_{c \nu, 2}(z)+\phi_{h \nu}(z)$ into Eq. (19) and integrating gives

$$
\frac{U^{(\mathrm{ex})} a^{2} N}{k_{B} T \mathcal{A} \bar{N}^{1 / 2}}=\frac{\chi N}{2}\left(\frac{w}{a N^{1 / 2}}\right),
$$

where $\bar{N} \equiv \rho^{2} a^{6} N$ is the invariant polymerization index of the diblock copolymer. Integrating Eq. (33) gives

$$
\begin{aligned}
-\frac{S_{c}^{(\mathrm{ex})} a^{2} N}{k_{B} \mathcal{A} \bar{N}^{1 / 2}}= & \frac{1}{3}\left(\frac{w}{a N^{1 / 2}}\right)^{-1}+\frac{1}{6}\left(\frac{w_{h A}}{a N^{1 / 2}}\right)^{-1} \\
& +\frac{1}{6}\left(\frac{w_{h B}}{a N^{1 / 2}}\right)^{-1}+\frac{\pi^{2}\left(h_{A}+h_{B}\right)^{3}}{4 a^{3} N^{3 / 2}} \\
& +\frac{\pi^{4} h_{A}}{64 f^{2} a N^{1 / 2}}\left(\frac{w_{h A}}{a N^{1 / 2}}\right)^{2} \\
& +\frac{\pi^{4} h_{B}}{64(1-f)^{2} a N^{1 / 2}}\left(\frac{w_{h B}}{a N^{1 / 2}}\right)^{2} .
\end{aligned}
$$

Although the integrals for the $A$ homopolymer entropy have to be integrated numerically, for the $B$ homopolymer we obtain

$$
\begin{aligned}
&-\frac{S_{h B, \text { trans }}^{(\mathrm{ex})} a^{2} N}{k_{B} \mathcal{A} \bar{N}^{1 / 2}}=-\frac{0.822467}{\alpha_{h B}}\left(\frac{w_{h B}}{a N^{1 / 2}}\right) \\
&+\frac{h_{B}}{\alpha_{h B} a N^{1 / 2}}\left(\ln \alpha_{h B}+1\right), \\
&-\frac{S_{h B, \text { conf }}^{(\mathrm{ex})} a^{2} N}{k_{B} \mathcal{A} \bar{N}^{1 / 2}}=\frac{1}{6}\left(\frac{w_{h B}}{a N^{1 / 2}}\right)^{-1} .
\end{aligned}
$$

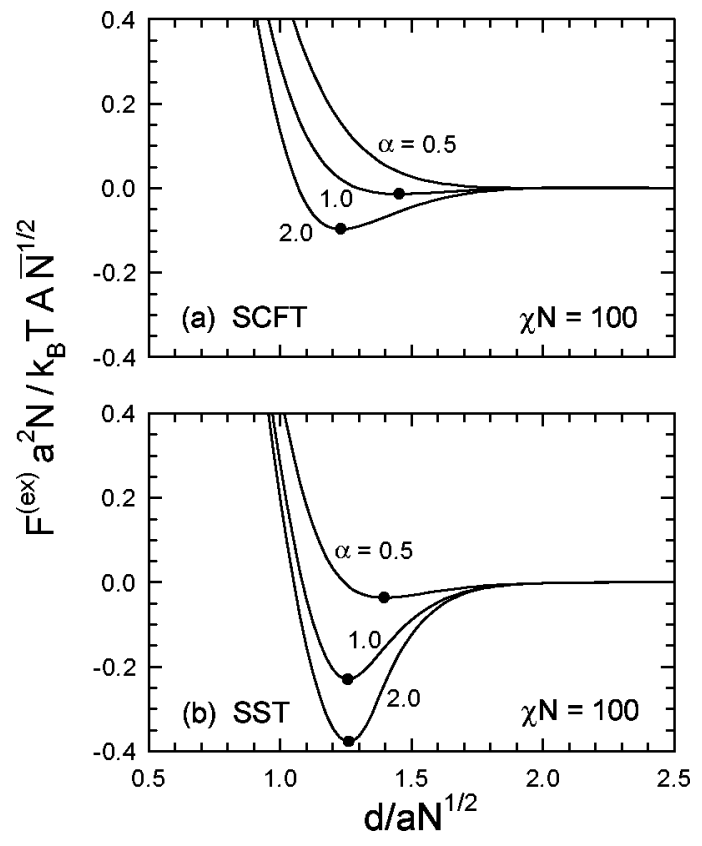

FIG. 2. Excess interfacial free energy, $F^{(\mathrm{ex})}$, as a function of separation, $d$, calculated at $\chi N=100$ for several molecular weight ratios, $\alpha$, using (a) SCFT and (b) SST. (Note that $\bar{N} \equiv \rho_{0}^{2} a^{6} N$ is the invariant polymerization index of the diblock copolymer.) The solid dots denote the position, $d_{\min }$, of minimum energy, $F_{\mathrm{min}}^{(\mathrm{ex})}$, for those curves displaying an attractive well.

The excess free energy, $F^{(\mathrm{ex})}$, can be minimized immediately with respect to $A / B$ interfacial width providing the result,

$$
\frac{w}{a N^{1 / 2}}=\frac{2}{(6 \chi N)^{1 / 2}} .
$$

Minimizing $F^{(\mathrm{ex})}$ with respect $h_{A}, h_{B}, w_{h A}$, and $w_{h B}$ under the constraints $h_{A} / h_{B}=f /(1-f)$ and $h_{A}+h_{B} \leqslant d$ has to then be done numerically. As before, the chemical potentials are adjusted so that the surface tension $\sigma$ is zero (i.e., so that $F^{(\mathrm{ex})} \rightarrow 0$ as $\left.d \rightarrow \infty\right)$.

\section{RESULTS}

Here, we present results for blends in which the diblock copolymers are symmetric (i.e., $f=0.5$ ), the homopolymers are of equal size (i.e., $\alpha_{h A}=\alpha_{h B} \equiv \alpha$ ), and the statistical segment lengths are the same (i.e., $a_{A}=a_{B} \equiv a$ ). Under these conditions, the $A$ - and $B$-rich homopolymer phases coexist at $\mu_{h A}=\mu_{h B}$. Furthermore, this symmetry means that the concentrations in the two homopolymer phases satisfy $\phi_{c}^{(A)}$ $=\phi_{c}^{(B)}, \phi_{h A}^{(A)}=\phi_{h B}^{(B)} \approx 1$, and $\phi_{h B}^{(A)}=\phi_{h A}^{(B)} \approx 0$.

Figure 2 shows the excess free energy $F^{(\mathrm{ex})}$ of two saturated monolayers as a function of their spacing $d$. The calculation is performed at $\chi N=100$ for several values of $\alpha$ using both SCFT and SST. In all cases, the interaction, which is given by the slope of $F^{(\mathrm{ex})}$, is negligible until the monolayers come into close proximity (i.e., $d \lesssim 2 a N^{1 / 2}$ ). If the homopolymers are large, then the monolayers initially experience an attraction until they come into close contact at which point a strong steric repulsion occurs. This produces attractive wells, where the depth, $-F_{\min }^{(\mathrm{ex})}$, and position, $d_{\min }$, are 

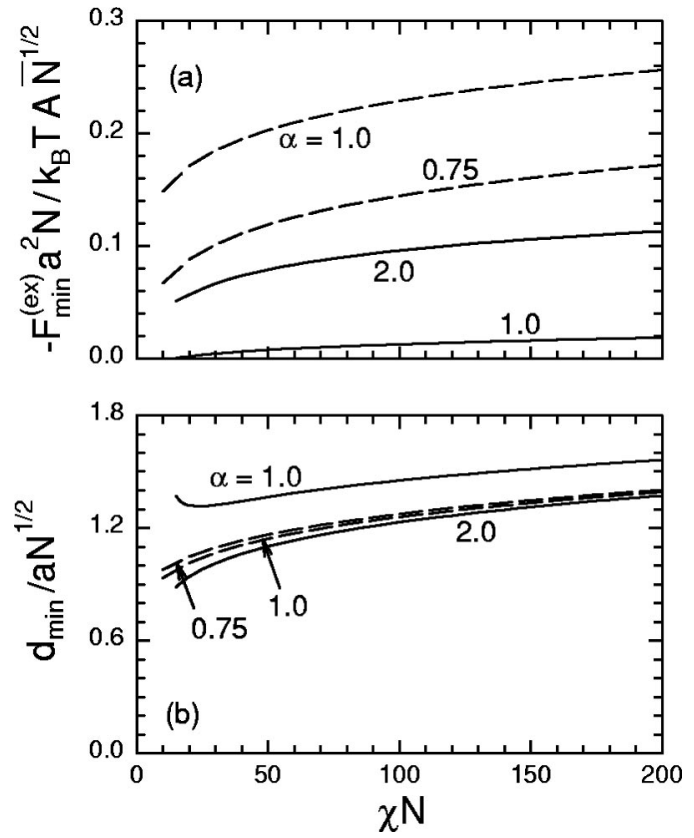

FIG. 3. (a) Depth, $-F_{\min }^{(\mathrm{ex})}$, and (b) position, $d_{\min }$, of the attractive well as a function of segregation, $\chi N$, for several molecular weight ratios, $\alpha$. (Note that $\bar{N} \equiv \rho_{0}^{2} a^{6} N$.) Solid and dashed curves denote SCFT and SST results, respectively.

indicated in Fig. 2 by solid dots. Although SST predicts a far stronger attraction than SCFT, it does produce the exact same qualitative trends. In particular, both theories predict that the attractive well fades as $\alpha$ decreases.

The depth, $-F_{\min }^{(\mathrm{ex})}$, and position, $d_{\min }$, of the attractive well is examined as a function of segregation $\chi N$ in Fig. 3. Naturally, the various energy contributions increase in magnitude as $\chi N$ increases, and thus the well becomes deeper. Furthermore, the copolymers stretch causing the monolayers to thicken, and consequently $d_{\text {min }}$ generally shifts to larger values as $\chi N$ increases. The effect of homopolymer molecular weight (i.e., $\alpha$ ) on the well is best demonstrated in Fig. 4. As $\alpha$ is reduced, the depth of the well decreases monotonically and its position moves out to $d=\infty$.

There exists a critical value of $\alpha$ at which point the well absolutely disappears, but locating it accurately is very difficult because the $-F_{\min }^{(\mathrm{ex})}$ versus $\alpha$ curves in Fig. 4(a) tail off very slowly. Regardless, the actual value is not important. The more relevant point is where the attraction becomes sufficiently small that the monolayer fluctuations prevent them from sticking together. However, a detailed calculation of this nature ${ }^{18}$ is beyond the scope of this paper. Instead, we construct tangents at the inflection points of the $-F_{\min }^{(\mathrm{ex})}$ versus $\alpha$ curves and extrapolate to the points indicated by diamonds in Fig. 4(a). The resulting value of $\alpha$ indicates where the depth of the attractive well becomes negligible. Based on this criterion, Fig. 5 divides the parameter space (i.e., $\chi N$ and $\alpha$ ) into two regions, one where there is a significant attraction and another where the well is either absent or at least negligible. The SCFT result (solid curve) is far more reliable than the SST prediction (dashed curve).

We can understand the source of the effective interaction between the monolayers by splitting the free energy into the
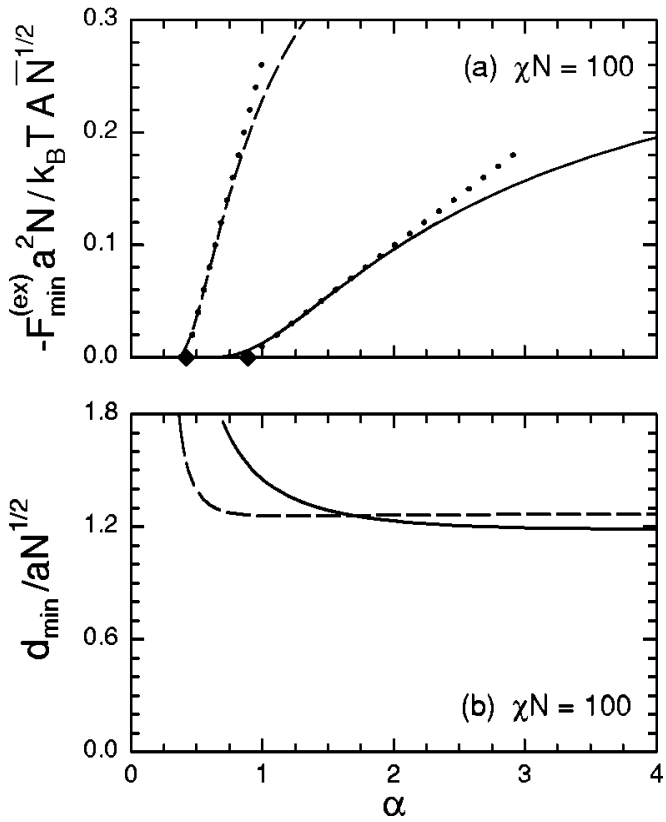

FIG. 4. (a) Depth, $-F_{\min }^{(\mathrm{ex})}$, and (b) position, $d_{\min }$, of the attractive well as a function of the molecular weight ratio, $\alpha$, calculated at $\chi N=100$. (Note that $\bar{N} \equiv \rho_{0}^{2} a^{6} N$.) Solid and dashed curves denote SCFT and SST results, respectively. The tangent construction in (a) is used to indicate the point where the attractive well becomes negligible.

various contributions described in Sec. II. However, in order to explain the behavior of these contributions, it is necessary to first examine the segment profiles as the monolayers are brought together. It is most useful to examine the $B$ segment profiles, because the overlapping of the $A$ copolymer brushes obscures some of the subtle effects. Figure 6 shows how the $B$ homopolymer $\phi_{h B}(z)$ and the $B$ copolymer $\phi_{c B}(z)$ profiles change as the monolayers are brought together. Plots are shown for $\alpha=0.5$ and 2.0. The profiles only change slightly as $d$ decreases from infinity to $1.4 a N^{1 / 2}$. It is interesting to note that the two values of $\alpha$ produce opposite effects; the monolayer thins slightly for $\alpha=0.5$ where as it thickens for $\alpha=2.0$. As the monolayers come into full contact leaving virtually no $A$ homopolymer between them, copolymer is forced out of the monolayer causing the brush thickness to rapidly decrease. Figure 6 also demonstrates that the low-

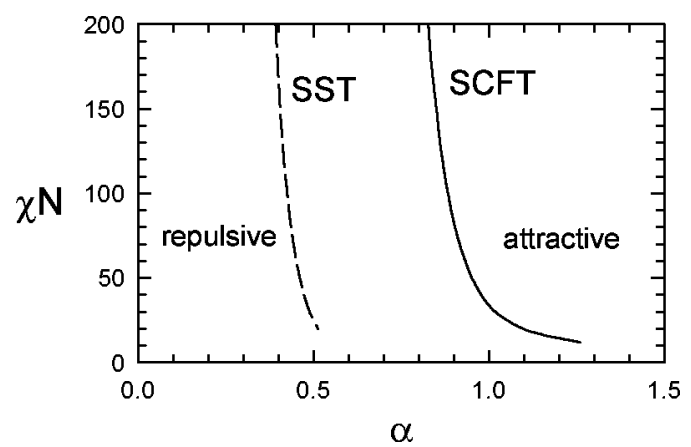

FIG. 5. Diagram separating parameter space (i.e., $\chi N$ and $\alpha$ ) into regions where the monolayer interaction has a significant "attractive" well and where the interaction is essentially "repulsive." The criterion used to separate these regions is demonstrated in Fig. 4(a). The solid and dashed curves denote SCFT and SST predictions, respectively. 

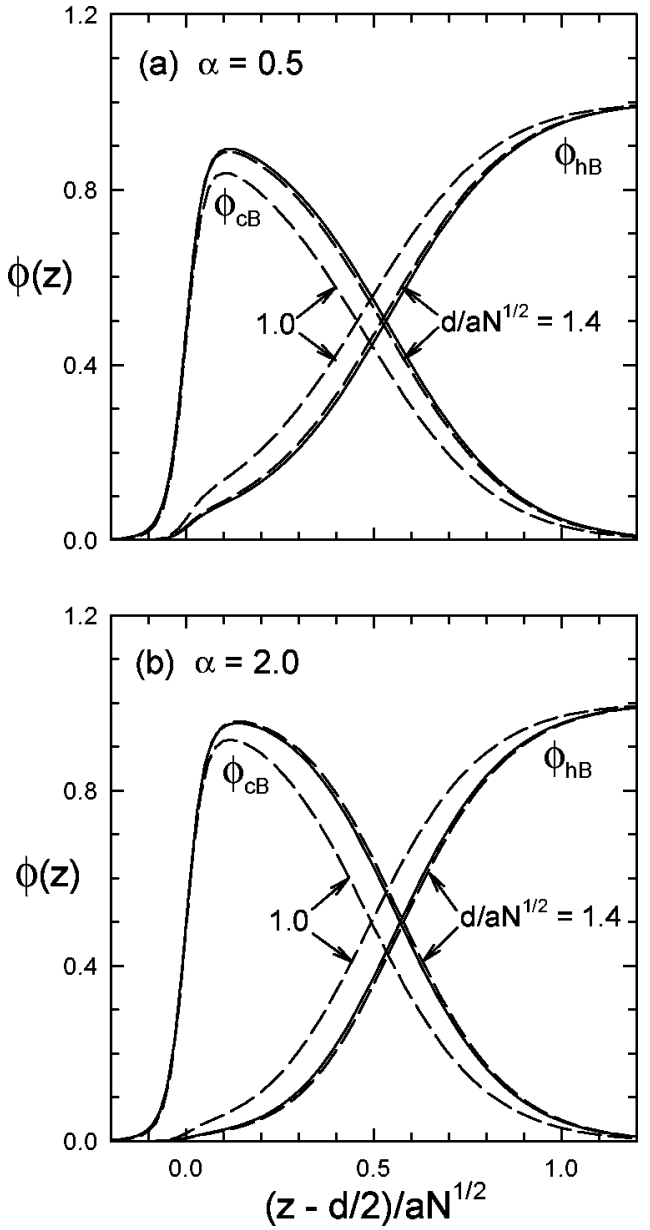

FIG. 6. Profiles at the $B$-rich copolymer/homopolymer interface calculated with SCFT at $\chi N=100$ for several monolayer spacings, $d$. (These curves are all shifted laterally so that the right $A / B$ interface occurs at zero.) Plots (a) and (b) are for $\alpha=0.5$ and 2.0, respectively. The dashed curves denote finite spacings, while the solid curves provide the $d=\infty$ limit. Notice the difference in the $d=1.4 a N^{1 / 2}$ profiles relative to the $d=\infty$ ones for the two values of $\alpha$.

molecular-weight homopolymer (i.e., $\alpha=0.5$ ) wets the copolymer brush to a slightly greater extent. It is also important to note that $\phi_{h B}(z)$ develops a steep gradient when it penetrates through to the A/B interface, which the assumed profile used in the SST calculation, Eq. (39), does not capture.

Now that we understand how the segment profiles behave as a function of separation $d$ and homopolymer molecular weight $\alpha$, it is reasonably straightforward to explain the various contributions to the excess free energy, $F^{(\mathrm{ex})}$. Figure 7 shows the seven contributions in Eqs. (18) and (25) each as a function of separation $d$. Curves are plotted for both $\alpha$ $=0.5$ and 2.0. The solid and dashed curves represent the SCFT and SST calculations, respectively. Although, there are significant quantitative differences, both theories generally predict the same qualitative behavior with the exception of a couple of instances, where the SST fails due to the problem mentioned above regarding the $\phi_{h B}(z)$ profile.

The excess internal energy, $U^{(\mathrm{ex})}$, plotted in Fig. 7(a) has a negligible effect on the interaction because it is nearly independent of the spacing $d$. In fact, SST assumes a constant $U^{(\mathrm{ex})}$ equal to that of a homopolymer/homopolymer
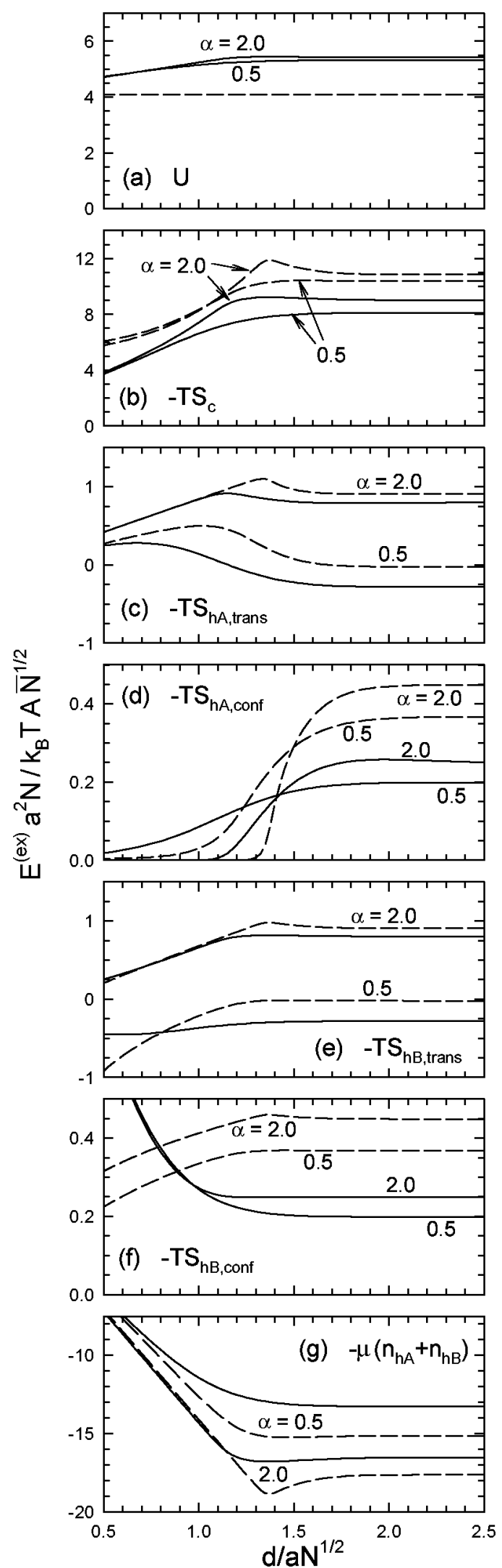

FIG. 7. The individual contributions to $F^{(\mathrm{ex})}$ defined in Eqs. (18) and (25) as a function of monolayer spacing, $d$, calculated at $\chi N=100$ for two different molecular weight ratios, $\alpha$. (Note that $\bar{N} \equiv \rho_{0}^{2} a^{6} N$.) The solid and dashed curves denote the SCFT and SST results, respectively. 
interface. ${ }^{14}$ Although, this is a reasonable approximation, the diblock monolayer will have a slightly wider interface due to the connectivity of the blocks. ${ }^{19}$ Consequently, the more accurate SCFT calculation predicts a higher value for $U^{(\mathrm{ex})}$. Note that in wet copolymer brushes, where more homopolymer penetrates to the $A / B$ interface, a sharper $A / B$ profile occurs resulting in a smaller $U^{(\mathrm{ex})}$. This is why the SCFT curve for $\alpha=0.5$ is slightly lower than that for $\alpha=2.0$. Similarly, $U^{(\mathrm{ex})}$ decreases at small spacings (i.e., $d \lesssim 1.2 a N^{1 / 2}$ ), because the narrower monolayers allow more homopolymer to reach the $A / B$ interface.

The excess entropic energy of the copolymer, $-T S_{c}^{(\text {ex })}$, is plotted in Fig. 7(b). To a large extent, these curves simply track the amount of excess copolymer at the interface. For example, reducing $\alpha$ causes more homopolymer to penetrate the monolayer, which lowers the amount of copolymer and thus $-T S_{c}^{(\mathrm{ex})}$ decreases. Similarly, the decrease in $-T S_{c}^{(\mathrm{ex})}$ as the monolayers are squeezed together is largely a result of the reduced copolymer content, although the reduction in stretching energy also contributes to this effect. Furthermore, the obvious peak in $-T S_{c}^{(\mathrm{ex})}$ for $\alpha$ $=2.0$ is a result of the monolayers thickening just before they come into full contact [see Fig. 6(b)].

The translational energy of the $A$ homopolymer, $-T S_{h A \text {,trans }}^{\text {(ex) }}$, shown in Fig. 7(c) favors swelling of the monolayers by the homopolymer. If the monolayers overlap, there is less room for the homopolymer and thus this contribution increases producing a repulsion. Because this energy is proportional to the number of molecules swelling the monolayer, the effective repulsion is magnified for small homopolymers. This is the same reason that low-molecularweight homopolymers have a stronger tendency to wet copolymer brushes as is evident in Fig. 6. At small $d$, after all the $A$ homopolymer is squeezed out from between the monolayers, $-T S_{h A \text {,trans }}^{(\mathrm{ex})}$ tends to zero.

The attractive component of the monolayer interaction originates from the configurational energy of the $A$ homopolymer, $-T S_{h A \text {, conf }}^{(\mathrm{ex})}$, shown in Fig. 7(d). When $A$ homopolymer is confined between the monolayers, it loses configurational entropy. High-molecular-weight homopolymers lose more entropy, because they produce drier brushes with sharper profiles. Nevertheless, this entropy is recovered when the homopolymer is squeezed out from between the monolayers, and thus $-T S_{h A \text {,conf }}^{\text {(ex) }}$ decreases producing an attractive interaction.

The excess translational energy, $-T S_{h B \text {,trans }}^{(\mathrm{ex})}$, of the $B$ homopolymer shown in Fig. 7(e) does not significantly affect the interaction between the monolayers. This is because it remains relatively uniform until the monolayers are in close contact, at which point it tends to zero. The SST calculation becomes inaccurate at these small separations, because the assumed profile for $\phi_{h B}(z)$ breaks down.

The excess configurational energy, $-T S_{h B \text {, conf }}^{(\mathrm{ex})}$, of the $B$ homopolymer shown in Fig. 7(f) also has no significant effect on the interaction. Only when the monolayers become tightly squeezed does $-T S_{h B \text {,conf }}^{(\text {ex }}$ show any significant variation. This occurs when the $B$ homopolymer starts penetrating the monolayer forming a sharp profile at the $A / B$ interface. Again the SST fails to capture this effect, because the as-

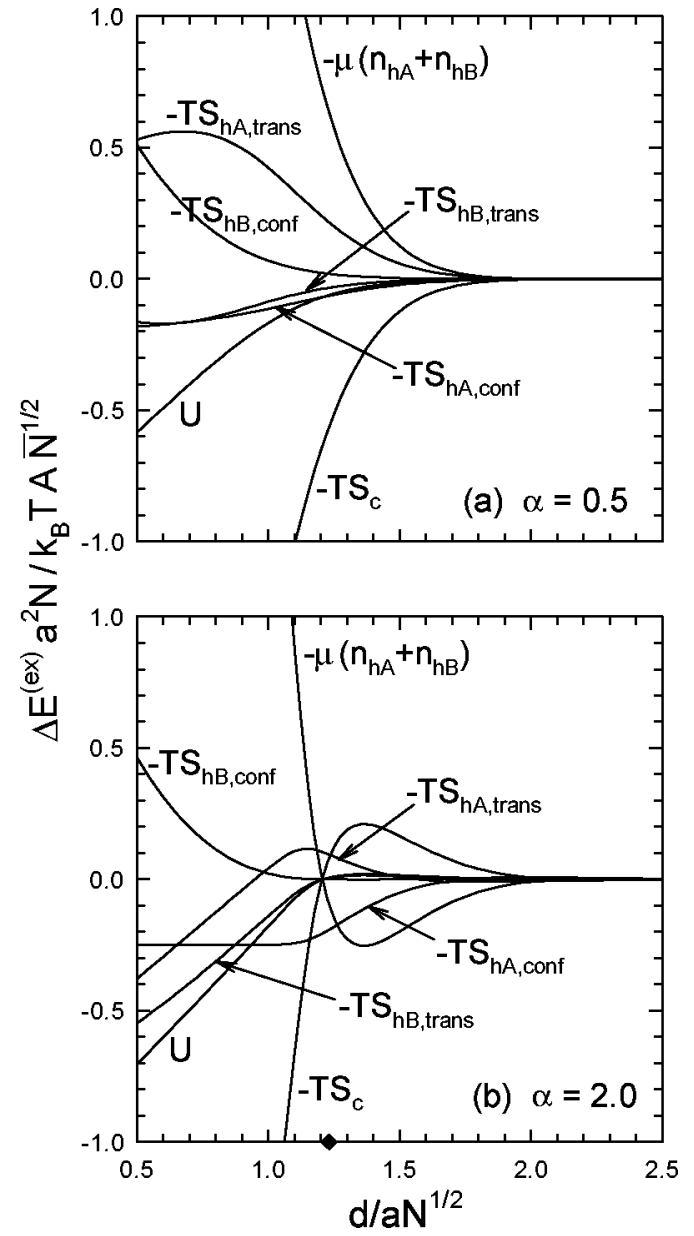

FIG. 8. The individual contributions to $F^{(\mathrm{ex})}$ plotted together as a function of spacing, $d$, calculated using SCFT at $\chi N=100$ for (a) $\alpha=0.5$ and (b) $\alpha=2.0$. (Note that $\bar{N} \equiv \rho_{0}^{2} a^{6} N$.) So as to compare the relative importance of these contributions, each curve is shifted vertically so that it becomes zero at large $d$. In (b), a solid diamond indicates the position, $d_{\min }$, of the attractive well.

sumed profile $\phi_{h B}(z)$ is unable to properly model this behavior.

The contribution responsible for the strong steric interaction occurring when the monolayers come into full contact is the excess chemical potential energy, $-\mu\left(n_{h A}^{(\mathrm{ex})}+n_{h B}^{(\mathrm{ex})}\right)$, shown in Fig. 7(g). This term is directly proportional to the excess copolymer at the interface. When the monolayers are squeezed together forcing copolymer to leave the monolayer, this energy term rises sharply producing the strong repulsion. Notice the small dip in the energy for $\alpha=2.0$ just before the brushes make full contact; this again reflects the slight thickening of the monolayers evident in Fig. 6(b).

Now that we have explained the behavior of the seven contributions to $F^{(\mathrm{ex})}$, it is important to examine their relative magnitudes. This is done in Fig. 8, where they are all plotted together and shifted vertically so that they become zero at large $d$. This figure only displays the SCFT results using separate graphs for $\alpha=0.5$ and 2.0. The two most significant terms, $-\mu\left(n_{h A}^{(\mathrm{ex})}+n_{h B}^{(\mathrm{ex})}\right)$ and $-T S_{c}^{(\mathrm{ex})}$, both track the excess copolymer concentration, and thus they have very similar shapes but with opposite signs. Since the chemical potential contribution is much larger in magnitude, these two 
terms combine to give a strong repulsion at small $d$, which we identify as the steric interaction. These two terms also contribute slightly to the attractive well for $\alpha=2.0$. The next two most important terms are due to the entropy of the $A$ homopolymer. The $-T S_{h A \text {,trans }}^{(\text {ex }}$ contribution produces a repulsion, which increases in strength as $\alpha$ decreases. On the other hand, $-T S_{h A \text {, conf }}^{(\mathrm{ex})}$ produces an attraction, which increases in strength as $\alpha$ increases. These two contributions are responsible for the interaction at intermediate distances where the monolayers start to come into contact, and it is their combined dependence on $\alpha$ that causes the crossover from an attractive well at large $\alpha$ to a totally repulsive interaction at small $\alpha$. The remaining three contributions, $U$, $-T S_{h B \text {, trans }}^{(\mathrm{e})}$, and $-T S_{h B \text {, conf }}^{(\mathrm{ex})}$, are relatively unimportant. This is because they are nearly uniform until $d$ becomes rather small. By that point, their effect is completely swamped by the strong steric interaction produced by $-\mu\left(n_{h A}^{(\mathrm{ex})}+n_{h B}^{(\mathrm{ex})}\right)$.

\section{DISCUSSION}

Of the two theories used here, SCFT is far more complete and thus more accurate. This is because SCFT is a first-principles approach which treats polymeric molecules realistically and automatically accounts for a wide range of detailed effects. To generate the more analytical SST requires a series of crude approximations. For example, it assumes the copolymer monolayers are highly stretched, which they are not. ${ }^{20}$ It also does not properly treat the connectivity of the copolymer blocks, and consequently it underestimates the $A / B$ interfacial width, ${ }^{19}$ which is why the SST prediction of $U^{(\mathrm{ex})}$ is inaccurate [see Fig. 7(a)]. Furthermore, SST ignores the translational entropy of the diblock copolymer end segments, ${ }^{20}$ and thus underestimates the width of the copolymer/homopolymer interface. This is largely why SST overestimates the configurational entropy of the homopolymers [see Figs. 7(d) and 7(f)], and thus predicts a stronger attraction than SCFT [see Fig. 2]. One danger with using SST is the possibility of ignoring important energy contributions. In fact, a previous SST calculation ${ }^{21}$ for micelles ignored the configurational entropy of the homopolymer, and consequently missed the attractive contribution to the micelle interaction. Despite the inaccuracies in SST, its simpler and more transparent expressions are conducive to generating intuitive explanations, which we now provide.

The optimum thickness of the monolayers is determined by a balance between the strong energetic tendency of copolymers to locate at the $A / B$ interface and the entropic penalty of stretching the copolymers so as to fill space uniformly. When the spacing $d$ between the monolayers becomes too small, the monolayers must narrow which forces some of the copolymer out into the bulk homopolymer-rich phases. The penalty for this, $-\mu\left(n_{h A}^{(\mathrm{ex})}\right.$ $+n_{h B}^{(\mathrm{ex})}$ ), produces the steric repulsion. This penalty is somewhat tempered by the fact the copolymers recover their stretching energy, $-T S_{c}^{(\mathrm{ex})}$, in the bulk phases.

At intermediate distances $d$, where the monolayers touch but are not forced to expel copolymer, the effective interaction originates from the entropy of the intervening ho- mopolymer. On one hand, its translational entropy, Eq. (34), favors a broad homopolymer distribution, $\phi_{h A}(z)$, which swells the monolayers. If the monolayers overlap, then there is less room for homopolymer causing this entropic energy to increase thus producing a repulsion. On the other hand, the configuration entropy, Eq. (35), is reduced when the homopolymer is confined between the two monolayers. To recover this entropy, the homopolymer tends to vacate the region between the monolayers in favor of a bulk homopolymer-rich phase. Since this can only happen if the monolayers come together, an effective attraction is produced. The crossover from a repulsive interaction at small $\alpha$ to an attractive interaction at large $\alpha$ (see Figs. 2, 4, and 5) is easily explained. The repulsive contribution from Eq. (34) increases as $\alpha$ decreases, where as the attractive contribution from Eq. (35) increases as the copolymer/homopolymer interface narrows, which occurs when $\alpha$ increases producing a drier monolayer.

We can now explain the curious effect in Fig. 6(b), where the monolayers initially experience a slight increase in thickness prior to the thinning that results when they start squeezing together. The configurational entropy loss that drives the homopolymer from between the monolayers producing the effective attraction at large $\alpha$ is also the source of this effect. Increasing the copolymer content and thus the thickness of the monolayers is just another way for the system to reduce the amount of homopolymer between the monolayers.

Bates and co-workers ${ }^{3}$ have recently demonstrated that under appropriate conditions, the symmetric ternary blends examined here can form bicontinuous microemulsion. The special significance of this phase is that it represents an absolutely stable blend, where the average domain size does not coarsen. However, to maintain a microscopic domain size, the monolayers must be reasonably flexible. ${ }^{9}$ Previous calculations ${ }^{8}$ have demonstrated that this requires small copolymer molecules. Of course, microemulsions are only valuable if they can compatiblize reasonable sized homopolymer molecules, which implies a large $\alpha$. However, $\alpha$ cannot be so large that a significant attraction occurs between the monolayers. As discussed in the Introduction, the monlayers would then collapse into a lamellar phase expelling the two homopolymers, which would then macrophase separate. This implies that the most useful microemulsions will be formed to the left of the solid curve in Fig. 5 at $\alpha \approx 0.8$. Thus, the previous experiments ${ }^{3,4}$ could potentially increase their homopolymer molecular weights by about four times without destroying their microemulsion.

By providing a detailed explanation for the effective interaction between monolayers, we are in a good position to suggest ways of improving polymeric microemulsions. In order to suppress the attractive interaction, which destroys blends with large $\alpha$, the configurational entropy loss of the homopolymer must be reduced. According to Eq. (35), this is accomplished by increasing the width of the copolymer/ homopolymer interfaces. Of course, an alternative copolymer architecture may improve the situation. ${ }^{2}$ However, we are presently examining an alternative solution of mixing large and small diblock copolymers. The polydispersity in 
the resulting copolymer monolayer should produce a more gradual interface and thus suppress the attraction. Furthermore, polydispersity is expected to decrease the stretching energy in the monolaye ${ }^{22}$ and thus increase its flexibility.

\section{CONCLUSIONS}

We have theoretically examined the effective interaction between two $A B$ diblock copolymer monolayers separated by a thin $A$-rich homopolymer domain with $B$-rich homopolymer phases on either side [see Fig. 1(a)]. This study was limited to symmetric blends, where the copolymer composition was $f=0.5$, the two homopolymers were of equal size (i.e., $\alpha_{h A}=\alpha_{h B} \equiv \alpha$ ), and the $A$ and $B$ statistical segment lengths were identical (i.e., $a_{A}=a_{B} \equiv a$ ). The accuracy of the self-consistent field theory (SCFT) allows us to reliably predict the optimum diblock copolymer size, while the simpler strong-segregation theory (SST) helps provide intuitive explanations for the interaction allowing us to suggest improved compatiblizers.

This study has provided a simple definitive explanation for the interaction between the monolayers. When the monolayers first come into contact, the interaction is controlled by the entropy of the $A$ homopolymer molecules between them. At small $\alpha$, the interaction is repulsive because homopolymer molecules are forced out of the overlapping monolayers causing a sizable reduction in their translational entropy. At large $\alpha$, an attraction occurs because the homopolymer molecules prefer to leave the confined region between the monolayers in order to increase their configurational entropy. At small $d$, where the monolayer thickness is forced to decrease, the interaction is dominated by the penalty of expelling copolymer from the interface, which produces a strong steric repulsion.

For the symmetric ternary blends examined here, our SCFT results suggest that the diblock copolymers should be slightly larger than the homopolymer molecules. If the diblock copolymers are too small, the resulting attraction between the monolayers will cause the blend to macrophase separate into a dry copolymer lamellar phase and two homopolymer-rich phases. On the other hand, the copolymer molecules should be as small as possible in order to produce the flexible monolayers ${ }^{8}$ necessary to have a stable microemulsion with reasonable sized domains. ${ }^{9}$

The SST results demonstrate that the attractive interaction can be suppressed by increasing the width of the copolymer/homopolymer profiles. We suggest that this can be accomplished by combining small and large diblock co- polymer compatiblizers. Not only will the polydispersity in the monolayer broaden the copolymer/homopolymer interface, it should reduce the stretching energy ${ }^{22}$ and thus increase flexibility. To obtain the optimum improvement in flexibility and reduced attraction, we are now performing calculations to determine the best size ratio of the two copolymers and the best composition of the monolayer. If all goes well, this simple solution could be an important step in producing commercially viable polymeric bicontinuous microemulsions.

\section{ACKNOWLEDGMENTS}

The authors are grateful to P. D. Olmsted for referring them to Ref. 18. The present work was supported by the Engineering and Physical Sciences Research Council (GR/ M18034).

${ }^{1}$ S. T. Milner and H. Xi, J. Rheol. 40, 663 (1996).

${ }^{2}$ R. A. L. Jones, Curr. Opin. Solid State Mater. Sci. 2, 673 (1997).

${ }^{3}$ F. S. Bates, W. W. Maurer, P. M. Lipic, M. A. Hillmyer, K. Almdal, K. Mortensen, G. H. Fredrickson, and T. P. Lodge, Phys. Rev. Lett. 79, 849 (1997)

${ }^{4}$ M. A. Hillmyer, W. W. Maurer, T. P. Lodge, F. S. Bates, and K. Almdal, J. Phys. Chem. B 103, 4814 (1999).

${ }^{5}$ H. S. Joen, J. H. Lee, N. P. Balsara, and M. C. Newstein, Macromolecules 31, 3340 (1998).

${ }^{6}$ C. W. Macosko, P. Guégan, and A. K. Khandpur, Macromolecules 29, 5590 (1996); K. Snødergaard and J. Lyngaae-Jørgenson, Polymer 37, 509 (1996); I. Fortelný, A. Zivný, and J. Juzza, J. Polym. Sci., Part B: Polym. Phys. 37, 181 (1999).

${ }^{7}$ A. M. Mayes, T. P. Russell, S. K. Satija, and C. F. Majkrzak, Macromolecules 25, 6523 (1992); K.-J. Jeon and R.-J. Roe, Macromolecules 27, 2439 (1994); H. S. Jeon, J. H. Lee, N. P. Balsara, B. Majumdar, L. J. Fetters, and A. Faldi, Macromolecules 30, 973 (1997).

${ }^{8}$ M. W. Matsen, J. Chem. Phys. 110, 4658 (1999).

${ }^{9}$ D. C. Morse, Phys. Rev. E 50, R2419 (1994).

${ }^{10}$ K. R. Shull, J. Chem. Phys. 94, 5723 (1991).

${ }^{11}$ A. N. Semenov, Macromolecules 26, 2273 (1993).

${ }^{12}$ D. H. Napper, Polymeric Stabilization of Colloidal Dispersions (Academic, London, 1983); in Observation, Prediction and Simulation of Phase Transitions in Complex Fluids, edited by M. Baus, L. F. Rull, and J.-P. Ryckaert (Kluwer, Dordrecht, The Netherlands, 1995).

${ }^{13}$ E. Helfand, J. Chem. Phys. 62, 999 (1975).

${ }^{14}$ A. N. Semenov, Sov. Phys. JETP 61, 733 (1985).

${ }^{15}$ M. W. Matsen, Phys. Rev. Lett. 74, 4225 (1995); Macromolecules 28, 5765 (1995).

${ }^{16}$ F. Schmid, J. Phys.: Condens. Matter 10, 8105 (1998).

${ }^{17}$ M. W. Matsen and F. S. Bates, J. Polym. Sci., Part B: Polym. Phys. 35, 945 (1997).

${ }^{18}$ S. T. Milner and D. Roux, J. Phys. I 2, 1741 (1992).

${ }^{19}$ A. N. Semenov, Macromolecules 26, 6617 (1993).

${ }^{20}$ M. W. Matsen and F. S. Bates, Macromolecules 28, 8884 (1995).

${ }^{21}$ L. Leibler and P. A. Pincus, Macromolecules 17, 2922 (1984).

${ }^{22}$ S. T. Milner, T. A. Witten, and M. E. Cates, Macromolecules 22, 853 (1989). 